\title{
Proposal of ZigBee Systems for the Provision of Location Information and Transmission of Sensor Data in Medical Welfare
}

\author{
Takefumi Hiraguri', Minoru Aoyagi', Yoshiaki Morino', Toshinari Akimoto1, \\ Kentaro Nishimori ${ }^{2}$, Tomomi Hiraguri ${ }^{3}$ \\ ${ }^{1}$ Faculty of Engineering, Nippon Institute of Technology, Miyashiro-machi, Saitama, Japan \\ ${ }^{2}$ Faculty of Engineering, Niigata University, Nishi-ku, Niigata, Japan \\ ${ }^{3}$ Faculty of Health Sciences, Tokoha University, Aoi-ku, Shizuoka-shi, Japan \\ Email: hira@nit.ac.jp
}

Received 30 August 2015; accepted 21 September 2015; published 25 September 2015

Copyright (C) 2015 by authors and Scientific Research Publishing Inc.

This work is licensed under the Creative Commons Attribution International License (CC BY). http://creativecommons.org/licenses/by/4.0/

(c) (i) Open Access

\section{Abstract}

This paper proposes a scheme to obtain location and vital health information using ZigBee system. ZigBee systems are wireless communication systems defined by IEEE 802.154. In the proposed scheme, location information is obtained using the Link Quality Indication (LQI) function of a ZigBee system, which represents the received signal strength. And, the vital health information are collected from the electrocardiogram monitor, the pulse and blood pressure device, attached to the patient's body. This information is then transmitted to an outside network by ZigBee systems. In this way, vital health information can be transmitted as ZigBee sensor data while patients with the ZigBee terminal are moving. In the experiments using actual ZigBee devices, the proposed scheme could obtain accurate location and vital health information from the sensor data. Moreover, to achieve high reliability in the actual service, the collected amount of sensor data was confirmed by the theoretic calculation, when a ZigBee terminal passed through ZigBee routers. These results indicate that the proposed scheme can be used to detect the accurate location of the ZigBee terminal. And over $99 \%$ of the sensor data on vital health information was obtained when the ZigBee terminal passed through approximately four ZigBee routers.

\section{Keywords}

ZigBee, Medical Welfare, Location Information, Sensor Data 


\section{Introduction}

In recent years, schemes for providing location information have been studied using various wireless systems. These systems intend to gather information, such as time, location, and specific actions. Moreover, wireless terminals require small-sized, lightweight, and power-saving devices, since the user carries these terminals. These systems typically use access point information of the cellular phone and global positioning system (GPS) system to provide monitoring services for children, prisons, etc. And these services have been developed aggressively and are being marketed [1] [2].

In the area of medical health, many different technologies have been combined together to improve healthcare monitoring and treatment. These include advancements such as sharing of electronic medical charts and managerial technique over a network, wireless body area networks (WBANs) that collect vital health information of a person for health care [3], and the telemedicine technology that helps diagnosis over the Internet.

This paper proposes the concept of system configuration and the scheme for the management of location/action history and health condition of patients and residents in a hospital or medical and welfare institution in order to provide comprehensive medical care. The proposed system consists of a terminal and multiple access points/routers for ZigBee as per the IEEE 802.154 standard [4]. The user carries a ZigBee terminal while moving around, and the location information is acquired from the received signal strength indicator (RSSI) obtained when a ZigBee terminal connects to the access point. Moreover, information about the body functions of the user (temperature, pulse, blood pressure, etc.) is simultaneously transmitted to the network as sensor data measuring several hundred kilobytes via the access points of ZigBee. Therefore, the proposed system can facilitate the management of action history and health conditions of patients and residents. However, in actual usage, the proposed scheme to obtain reliable location and vital health information must be developed by considering changes in the propagation environment [5].

In this study, the communication area of ZigBee terminal is optimally controlled, and hence, the location information will be reliable. ZigBee terminal is controlled in order to always connect with one access point. Therefore, transmitting power is adjusted so that the signal should not reach two access points or more. We experimentally confirmed the effectiveness of the proposed scheme. Moreover, the transmitted data volume within the communication area was analyzed by each street course.

The remainder of this paper is organized as follows. In Section 2, we describe issues in conventional schemes. Section 3 proposes the system to obtain location and vital health information. In Section 4, we evaluate the proposed scheme.

\section{Conventional Systems}

Various wireless technologies that obtain location information have been proposed. For example, radio frequency-identification (RF-ID) [6]-[9] and sensor systems using wireless LAN (Wi-Fi) [8] [9] are well-known conventional wireless technologies. RF-ID is effective for short-range communication owing to the small size of the hardware and the power savings achieved. The limitation of RF-ID is that it can transmit only a slight amount of data at a time. Hence, it does not suit the purpose of this study, because this study aims to transmit data about health conditions etc. Latest Wi-Fi systems incorporate a power saving module, and the transmission link rate is also high. However, the installation location is limited because the access points require a cable for power supply. As for the rest, although the GPS [10] [11] is a popular outdoor location information system, it cannot work in indoor scenarios. Moreover, GPS does not allow exchanging health condition data.

The other hand, ZigBee systems are more suited to the purpose of this study than the techniques described above. ZigBee is defined in IEEE 802.154 standard and can be easily utilized for commercial purposes. Although transmission link rate is low, it enables transmission of a constant amount of data. Further, there is good inter-connectivity between ZigBee modules and low-cost modules, and its battery will achieve a long operating life. The proposed scheme effectively utilizes these features of ZigBee. Section 3 describes the details of the ZigBee system.

In order to collect health-related information from patients and residents, the WBAN system that uses a device including a radio transmitter attached to the body has received increasing attention, and this system is standardized in IEEE 802.15.6 [12] and [3]. It is used to collect the health information of a person for medical treatment and nursing care. Therefore, the WBAN is a special-purpose sensor network designed to operate autonomously to connect various medical sensors and appliances, located inside and outside the human body. 
However, since the WBAN system is developed to work in very short distance ranges, another system that can transmit in the distance range of 10 - 20 meters and has a mobility function to transmit the collected health information to an outside network is necessary. Hence, the WBAN can be used to collect health information from the human body, and ZigBee can be used to transmit this information to an outside network and to obtain the user's location information, as shown in Figure 1. Therefore, using ZigBee together with a WBAN provides an effective service.

This paper proposes a system to enable data transmission in the outside network using a ZigBee system, and a scheme to obtain location and health information.

\section{Proposed System and Scheme}

We proposed ZigBee system to obtain accurate location and vital health information in our previous work [17]. This system is configured as shown in Figure 2. An End Device (ZED) as a terminal is connected to the multiple ZigBee Routers (ZRs) as access points. The terminal ID and Link Quality Indication (LQI) information are obtained from ZR when the ZED connected to the ZRs. The information are sent to the ZigBee Coordinator (ZC). ZC has functions to control the ZR intensively. The LQI indicates the received power of signals as RSSI

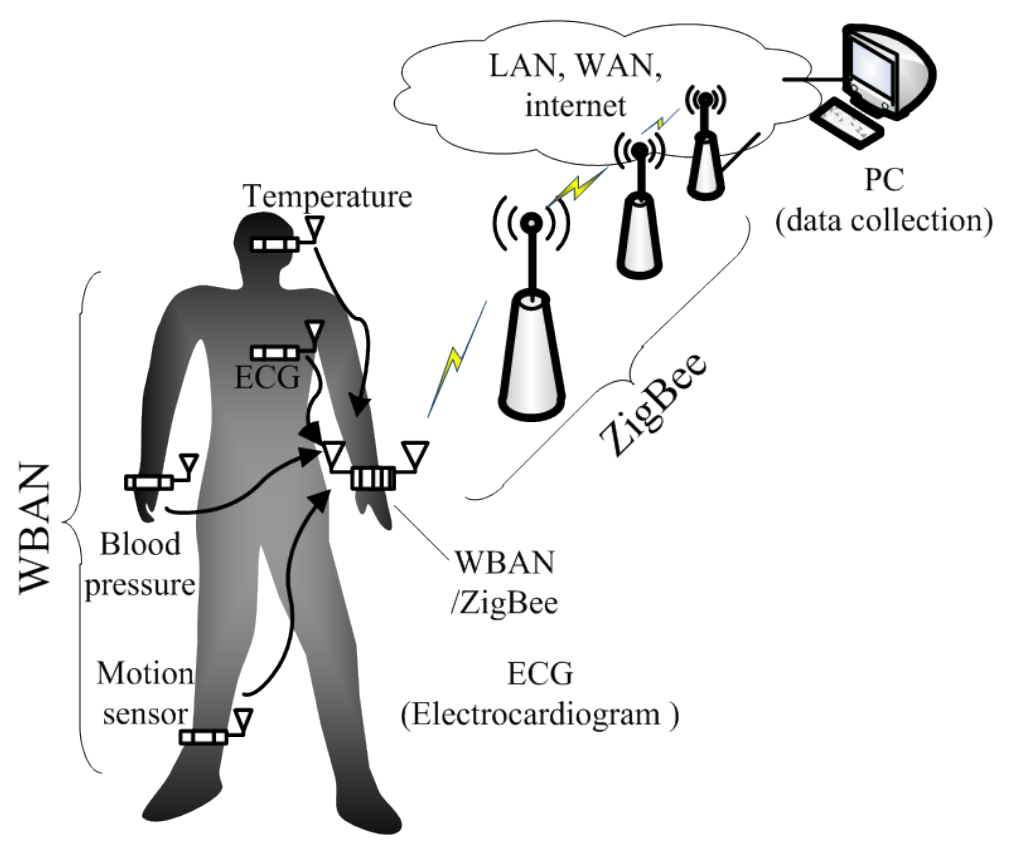

Figure 1. Health condition monitor service using WBAN and ZigBee.

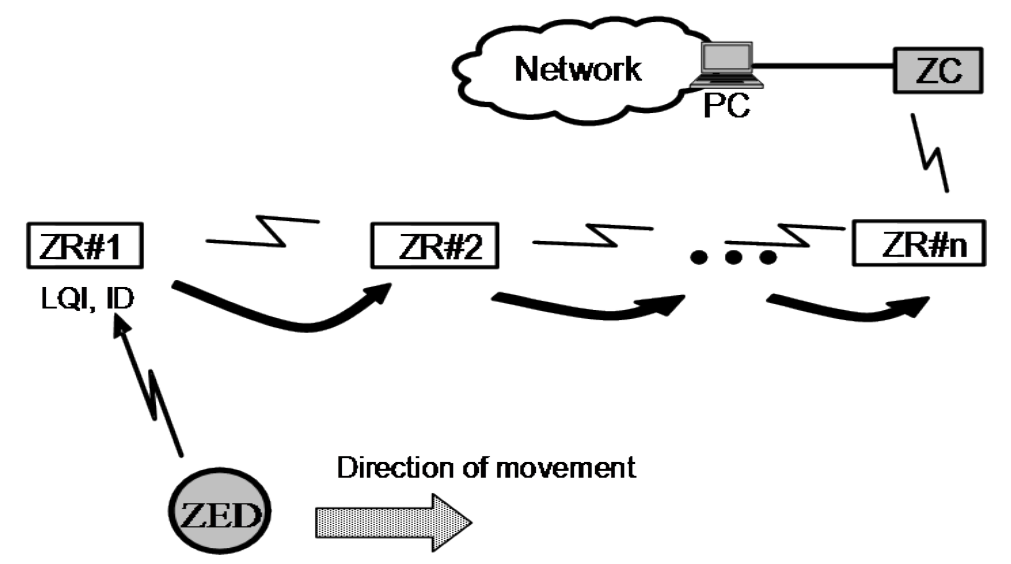

Figure 2. Network configuration using ZigBee. 
from ZED. The LQI is set to the normalizing value from 1 to 255 [6]. The ZC manages the location information and the migration pathway based on the location information, which is saved on a personal computer in the backbone networks. " 1 " is set if the ZED approaches a ZR. " 0 " is set if the ZED moves away from a ZR. In the proposed scheme, " 1 " or "0" is set according to the level of the LQI. These values are managed in the time specific to each ZR. Therefore, the movement history of the user is measured by this scheme if ZR is configured in advance at a pre-determined location. However, it is difficult to determine the threshold level of LQI, since the propagation environment changes by factors such as multipath signals and shadowing effect etc., especially in indoor environment. These factors may lead to erroneous determination of the ZR that is nearest to the ZED. This issue is addressed by controlling the communication area of a ZED such that the ZED can connect to the nearest ZR. In Figure 3, the radius of the communication area of the ZED is $r$ [m]. And the ZED communicates with the nearest ZR. This communication area $r$ is controlled to outside the range of ZR2. Therefore, the transmission power in the ZED is adjusted in order to narrow small communication area.

The ZED may not connect to the ZR with which it wishes to communicate when the communication area is extremely small. Moreover, the ZED transmits sensor data of vital health information. Hence, the transmission of health information is impossible if the area is extremely small. The walking speed of a human is $v[\mathrm{~m} / \mathrm{s}]$ and the transmission link rate is $R_{L}[\mathrm{bit} / \mathrm{s}]$. In Figure 4, when the ZR passes the center in the communication area of the ZED, the volume of sensor data of vital health information from ZED is represented as

$$
C=\frac{2 r R_{L}}{8 v}
$$

where the volume of sensor data is $C$ [Kbyte]. For example, $c$ is the data volume, and this is approximately 300 Kbyte if the walking speed of a human is $v=1.0 \mathrm{~m} / \mathrm{s}$, the radius of the communication area is $r=5 \mathrm{~m}$ and the ZigBee link transmission rate is $R_{L}=250 \mathrm{Kbit} / \mathrm{s}$. This transmission link rate is defined in IEEE 802.154 standard. In Reference [13]-[15], the walking speed is described as about 0.5 to $2.3 \mathrm{~m} / \mathrm{s}$. Walking speed was $v=1.0 \mathrm{~m} / \mathrm{s}$ as average value in Reference [13]-[15].

The communication area radius from transmission power is calculated as follows:

$$
P_{t}=P_{r}-\left(G_{t}-L_{p}-G_{r}\right)
$$

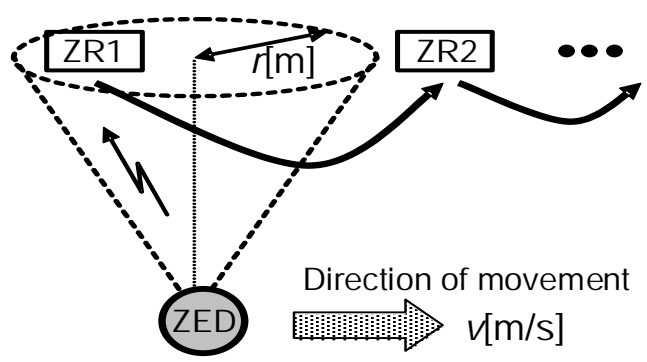

Figure 3. Movement of ZED and ZR.

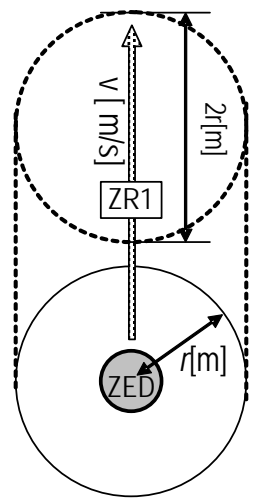

Figure 4. Movement communication area of ZED. 


$$
L_{p}=10 \log _{10}\left(\frac{4 \pi r}{\lambda}\right)^{n}
$$

$P_{t}$ is the transmisson power $[\mathrm{dBm}], P_{r}$ is the received power $[\mathrm{dBm}], G_{t} / G_{r}[\mathrm{dBi}]$ is the antenna gain at the transmitter or receiver side, and $L_{p}[\mathrm{~dB}]$ is the propagation path loss. $P_{t}$ is determined by between $P_{r}$ and $r$ because the reception sensitivity is $-97 \mathrm{dBm}$ in this system. $G_{t}$ and $G_{r}$ are $2 \mathrm{dBi} . L_{p}$ is calculated from the radius of the communication area and $\lambda=1$ /frequency: $2.4 \mathrm{GHz}$. The path loss coefficient $n$ is 3 , since this scheme is assumed to be indoors [16]. We obtain plots of the transmission power $\left(P_{t}\right)$ and sensor data volume $(c)$ versus the radius of the communication area $r$ from Equations (1) to (3) as shown in Figure 5. The unit “dBm” is converted to "mW" for greater understanding. We assumed the amount of sensor data for vital health information to be 300 Kbyte in one communication area. In Figure 5, this situation corresponds to a communication area radius of $5 \mathrm{~m}$. The transmission power is $0.01 \mathrm{~mW}$ and $P_{t}$ is controlled by this value. The experiment results are obtained by our previous work [17] is described in Subsection 4.1.

\section{Evaluation of Proposed Scheme}

In this section, we evaluate the proposed scheme experimentally and theoretically. Subsection 4.1 presents an experimental evaluation of location information and action history by our previous work [17]. Subsection 4.2 provides a theoretical calculation for the amount of sensor data in the ZigBee communication area.

\subsection{Experimental Evaluation of Location Information and Action History}

We experimentally demonstrated the performance of the proposed scheme. The experimental setup consisted of three ZRs and a moving ZED terminal. The route of the ZED is shown in Figure 6. The specification of the ZigBee module (IMI-Z-M-001) [7] is shown in Table 1. The reception sensitivity of the RSSI is $-97 \mathrm{dBm}$ in this module. The ZED and ZRs used in the experiment are shown in Figure 7. ZigBee device put in case, and horizontal and vertical sizes are about $50 \times 70 \mathrm{~mm}$ respectively.

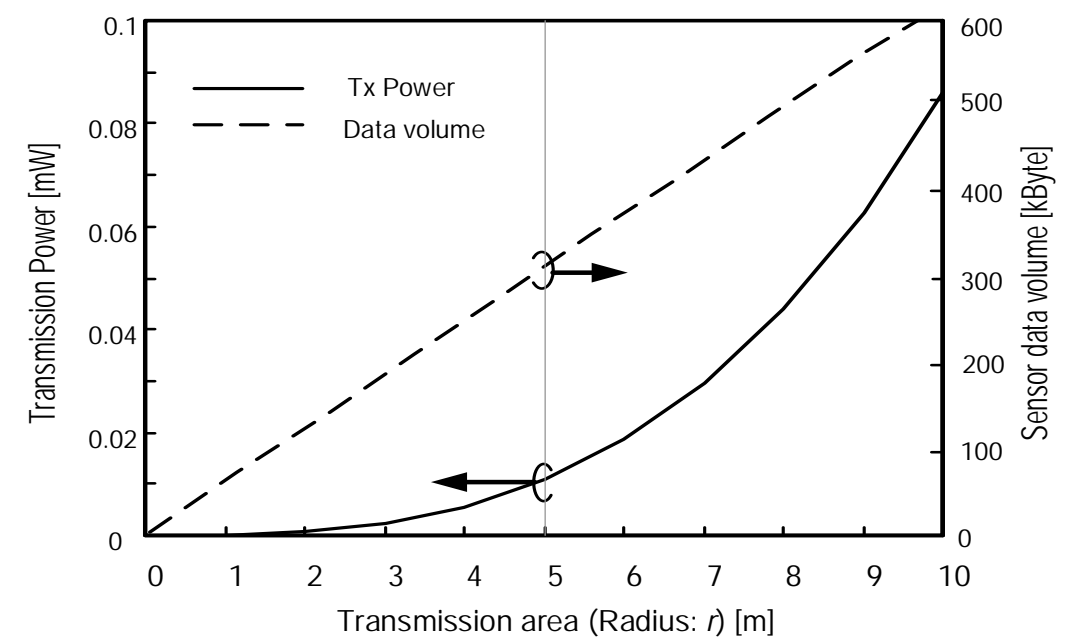

Figure 5. Transmission power and data volume versus radius $r$.

Table 1. IMI-Z-M-001 module specification.

Reception sensitivity

Transmission power

Link rate
$-97 \mathrm{dBm}$

$1 \mathrm{~mW}$ (Default)

$0.01 \mathrm{~mW}$ (w/Area control) 


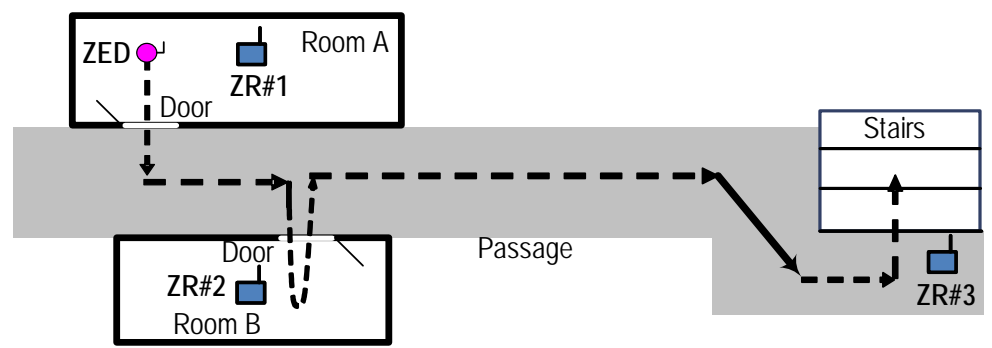

Figure 6. Route of the ZED in our experiment.

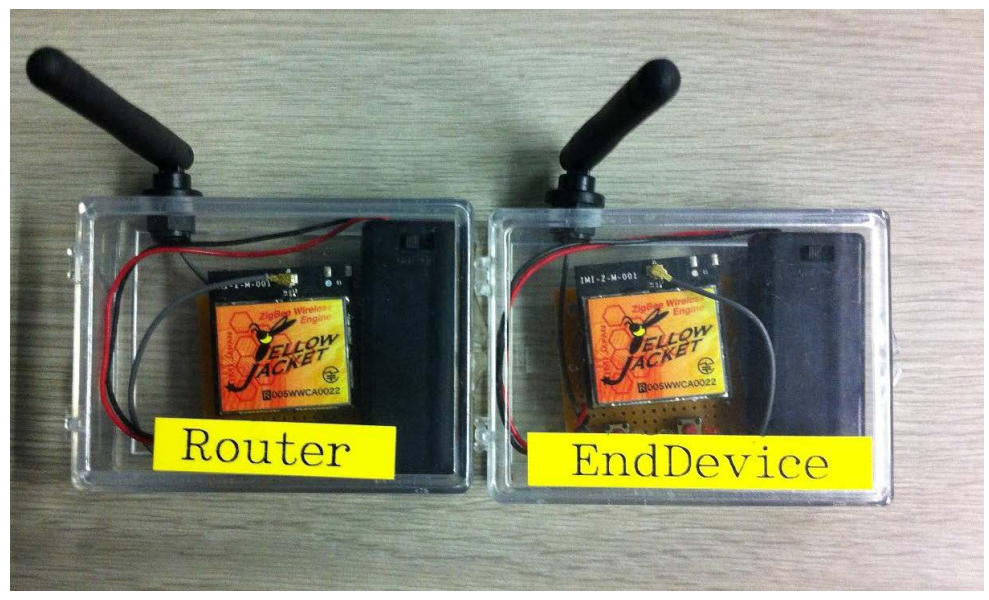

Figure 7. Images of the ZED and the ZR devices (IMI-Z-M-001).

The ZED terminal holder was moved in Rooms A and B and along the stairs, as shown in Figure 6. ZR\#1 and ZR\#2 were located inside Rooms A and B, respectively. ZR\#3 was positioned in front of the stairs. The ZED terminal holder entered Room B after leaving Room A. Next, the ZED terminal holder moved toward the stairs. The measured value of the LQI is shown in Figure 8. The solid lines in the figure represent the measurement results with area control with respect to time. The dotted lines indicate the results obtained by using a default value of the transmitting power $(1 \mathrm{~mW})$ without area control. Using area control, the power transmitted by the proposed scheme became $0.01 \mathrm{~mW}$, except at the target point. In Figure 8, first, ZR\#1 was set to "1" because the LQI of ZR\#1 is higher than that of the other ZR. Next, ZR\#2 was set to "1" because its LQI increased considerably. From these evaluation results, the determined pattern indicates that the ZED terminal holder moved from Rooms A to B. Finally, the LQI of ZR\#3 increased, and ZR\#3 was set to "1". In this result, the ZED terminal holder moved from Room B to the stairs. When the values "1" or "0" in ZR\#1, ZR\#2, and ZR\#3 were recorded, the pattern [100], [010], and [001] was obtained using the proposed scheme. This pattern represents a route from Rooms A to B, and Room B to the stairs. If some patterns are pre-generated, the system administrator may easily determine the route via pattern matching. From these evaluation results, the approximate location information and movement history of the ZED terminal holder can determined by changing the value of LQI.

However, when a default value of the transmitting power is used without area control, it is difficult to obtain accurate location information because the change in the LQI value is unclear. Moreover, in a scheme without any area control, it may not be possible to specify the ZR nearest to the ZED terminal holder since the ZED is connected to multiple ZRs. On the other hand, when a transmitting power of $0.01 \mathrm{~mW}$ with area control is used, the change in the LQI value is clear, although the level of LQI is lower. From the experimental results, it is confirmed that the proposed scheme provides more accurate operation when the area radius is optimized.

In the proposed scheme, the ZED can clearly detect only the ZR in the line-of-sight because the communication area becomes limited by lowering the transmission power of the ZED. Moreover, the multi-pass signal from the ZR barely transmits to the ZR beyond the line-of-sight. The conventional scheme does not control transmission power, and therefore, the signal from the ZED will reach two or more ZRs. Therefore, in this case, the position of the ZED may not be clearly specified. We can say that the results in Figure 8 are reliable because the 


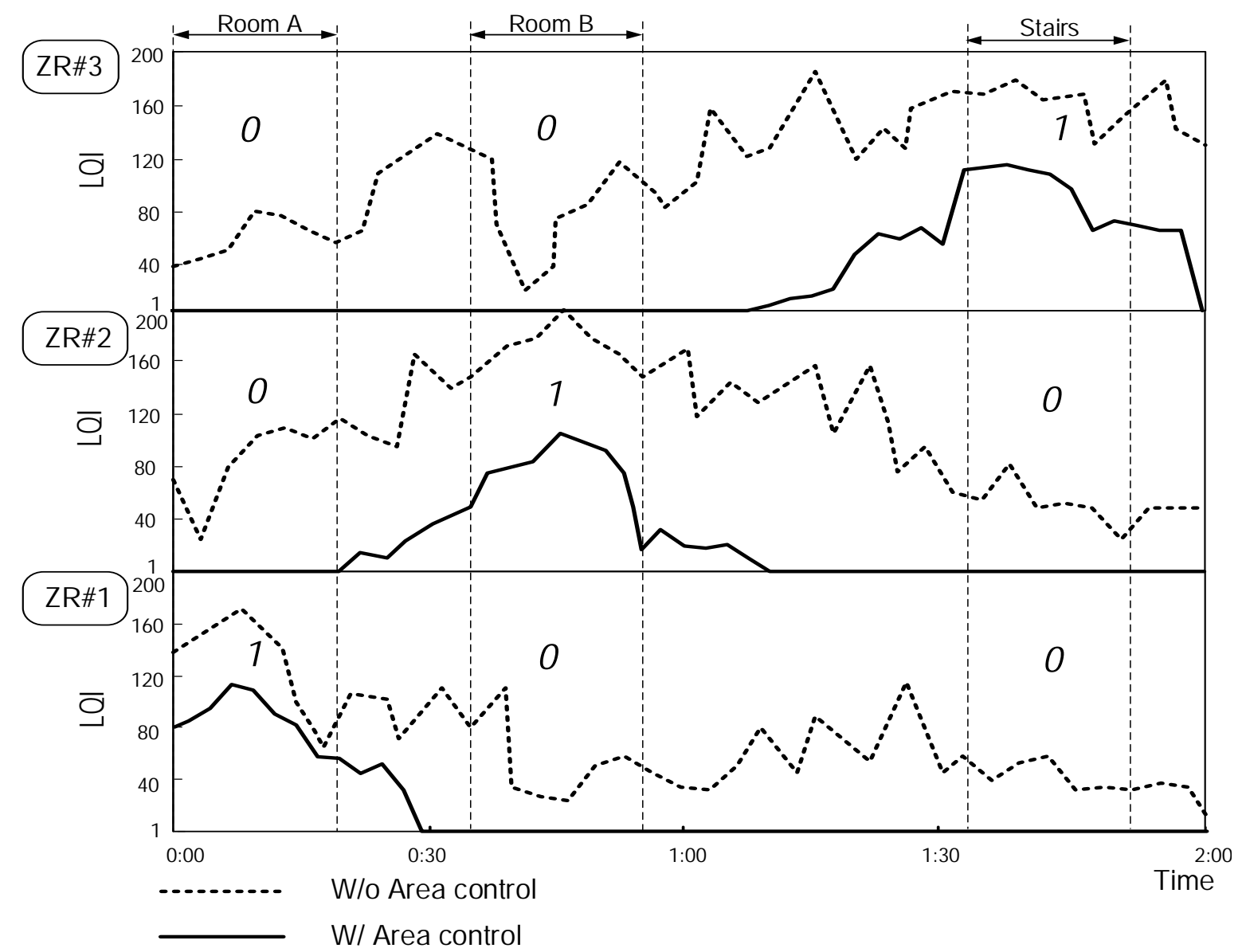

Figure 8. Evaluation result of the LQI value versus time in our experiment

same results are obtained when this experiment is repeated 10 times. However, these results are achieved for a specific route, and hence, other experiment routes may not yield equally good results. In order to limit the communication area of the ZED, the installation location of the ZR may have to be selected carefully for other routes. Moreover, in Equation (1) of the theoretical calculation, the sensor data volume was estimated when the ZR passed the center of the communication area. In the real service environment, a different route that does not pass the center may be taken. The next Subsection 4.2 presents a theoretical analysis of the sensor data volume when the ZR passes various routes within the communication area.

\subsection{Analysis of Sensor Data Amount and Different Routes in the Communication Area}

Various ZR routes pass the communication area of ZED, and these routes can be very complex because patients carry around the ZED device. Hence, for a simple evaluation analysis, we assumed that the ZED passes a straight-line route. The outline for the analysis of a ZR passing the communication area of the ZED is shown in Figure 9. In this figure, the ZED passes straight. This straight-line route changes slightly. The transit time of the $\mathrm{ZR}$ in the ZED communication area is calculated from this figure using the following equations:

$$
\begin{gathered}
T=\frac{L \times 2}{v} \\
L=\sqrt{r^{2}-R^{2}} \\
R=\Delta r \times n(0 \leq R \leq r, n=0,1,2,3, \cdots)
\end{gathered}
$$

where $T$ is the transit time [sec] taken to pass the area in Equation (4), and $L[\mathrm{~m}]$ is half the distance through the 


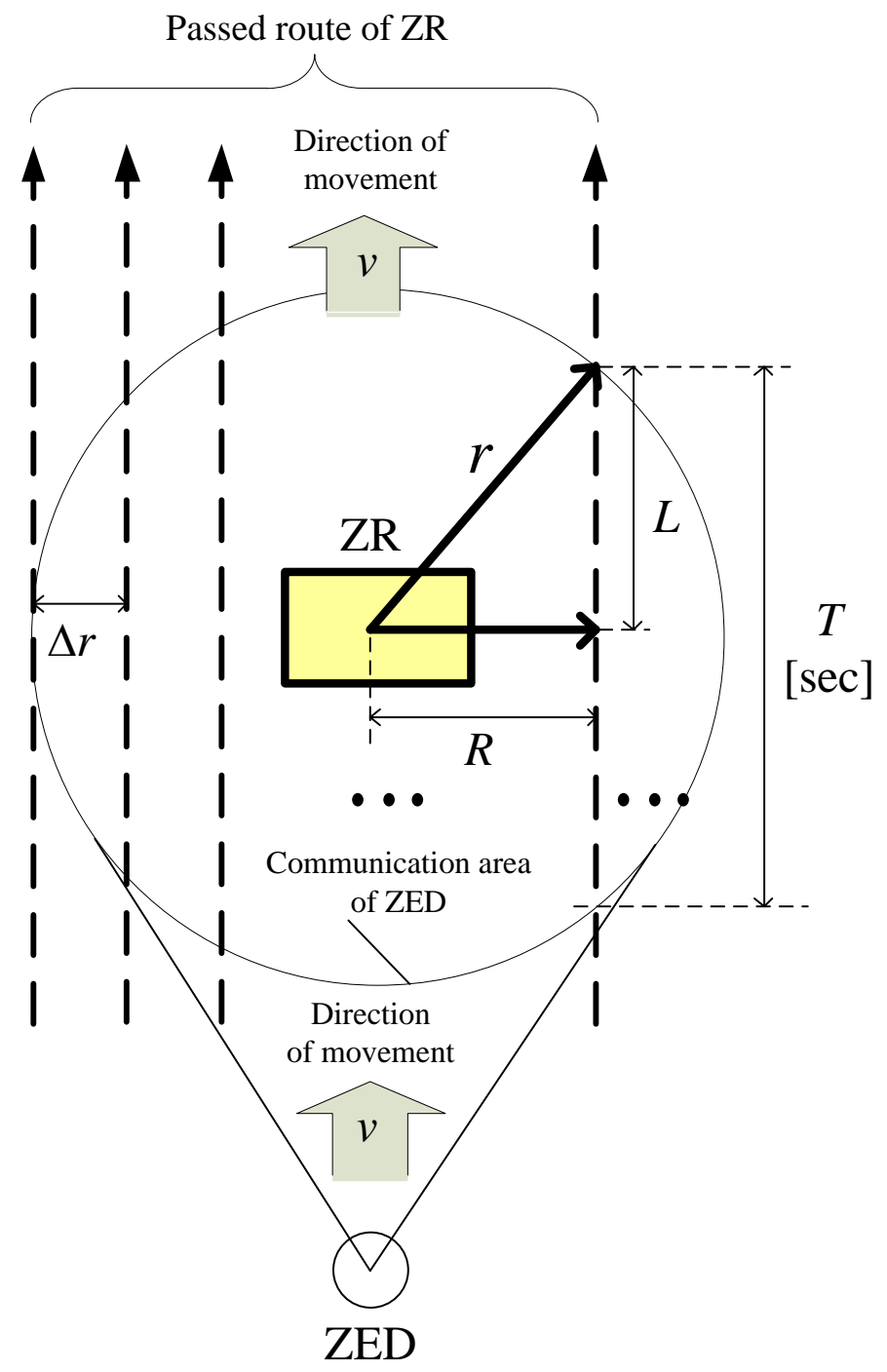

Figure 9. Transmission time analysis for different routes.

ZED communication area. The moving speed of the ZED is $v[\mathrm{~m} / \mathrm{s}] . R[\mathrm{~m}]$ is the distance from the center when passing the communication area at intervals of $\Delta \mathrm{r}$ [m]. Therefore, $\Delta r$ is set to $0.01 \mathrm{~m}$, and $n$ is the number of routes through which the ZR passed the ZED communication area.

However, the sensor data may not be transmitted throughout the transit time $T$, since the overhead of packets and the procedure time for connection are spent by ZigBee communication. The useless time is the time taken to establish a connection between the ZED and ZC, the time to transmit the header of a data packet, and the carrier sense (CS) time at each packet transmission, as shown in Figure 10. In this figure, route request (RREQ) and route reply (RREP) are the management frames for a connection from the ZED to the ZC, and this time is $\alpha$ [sec] (ZRs, ZC, and ZED are ten hops). $\beta$ [sec] is the sum of the carrier sense time and the time taken to transmit the header of each packet. Therefore, this value is the overhead time to have pulled out the transfer time of the data payload. The data payload is 112 bytes, and the value of each parameter is listed in Table 2. The time available for transmitting the sensor data is calculated using the following equations:

$$
T_{r}=T-(\alpha+\beta)
$$

$$
T_{c}=\frac{\text { Data Volume }}{\text { Data Payload }} \times \text { Frame Length }
$$




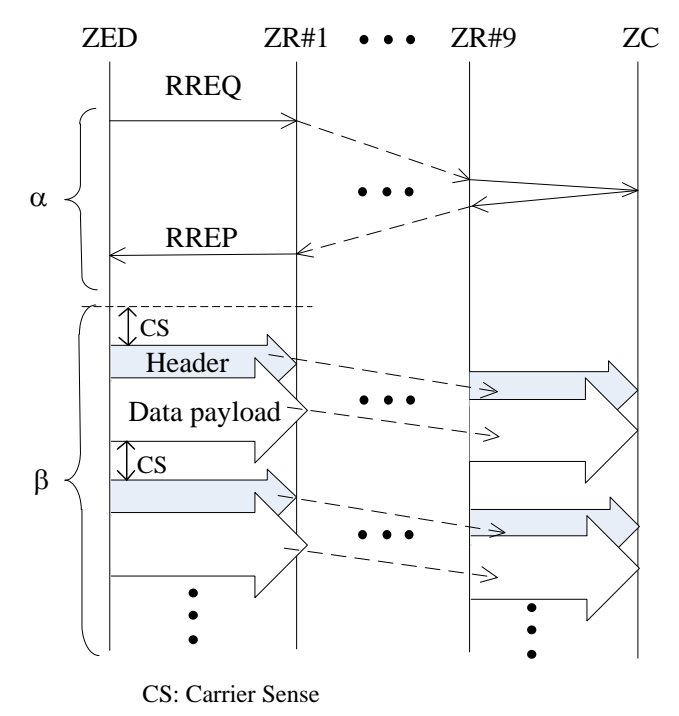

Figure 10. Connection sequence and overhead in a 10-hop network.

Table 2. Value of overhed and sequence procedure time.

\begin{tabular}{cccc}
\hline \multicolumn{2}{c}{ Elements } & Size & Spent time \\
\hline$\alpha$ & RREQ & & $0.96[\mathrm{msec}] \times$ hop counts \\
$\beta$ & RREP & 15 Byte & $0.672[\mathrm{msec}] \times$ Number of transmission \\
& Header & 21 Byte & $0.128[\mathrm{msec}] \times$ Number of transmission \\
\hline
\end{tabular}

$T_{r}$ [sec] is the time taken to transmit sensor data. The data payload transmission time except the overhead is calculated by Equation (8); $T_{c}$ [sec] is the total time taken for data payload transmission, and DataVolume [byte] is the actual transmitted information volume (sensor data volume). DataPayload [byte] is 112 Bytes, and Frame Length [sec] is the transmission time for one data payload. Therefore, FrameLength is (112 Byte $\times 8$ )/250 Kbit/s (ZigBee link rate). In Equations (7) and (8), the desired information volume may be entirely transmitted in the communication area if $T_{r}$ is larger than $T_{c}$. Moreover, when the ZR and ZED pass various routes, the probability that the sensor data sent from the ZED are received at the ZR is calculated by the following equations:

$$
\begin{gathered}
S=\frac{N_{\text {succ }}}{N_{\text {all }}} \times 100[\%] \\
T_{r}-T_{c} \geq 0 \text { (Completely transmitted) } \\
T_{r}-T_{c}<0 \text { (Faikure) }
\end{gathered}
$$

$S$ is the probability, and $N_{\text {succ }}$ is the number of routes through which sensor data is entirely transmitted in the communication area according to the condition in Equation (10). $N_{\text {all }}$ is the number of all routes that the ZR passed in the communication area. Therefore, it indicates $n$ in Equation (6).

The probability against the communication area is evaluated using these equations, as shown in Figure 11. In this figure, Data volume is 250 Kbyte, and the moving speed of the ZED ranges from $0.6-1.4 \mathrm{~m} / \mathrm{s}$. A probability of $70 \%$ is shown in Figure 11 for a speed $v$ of $1.0 \mathrm{~m} / \mathrm{s}$ and an area radius $r$ of $5 \mathrm{~m}$. However, as indicated by these results, a probability close to $100 \%$ is not obtained by passing a single ZR. Hence, the ZED should pass through two or more ZRs. The number of ZRs to be passed through to obtain a probability of almost $100 \%$ was evaluated, as shown in Figure 12. A data volume of 250 Kbyte ensured about 99\% probability when the ZED passed through four ZRs at $1.0 \mathrm{~m} / \mathrm{s}$. 


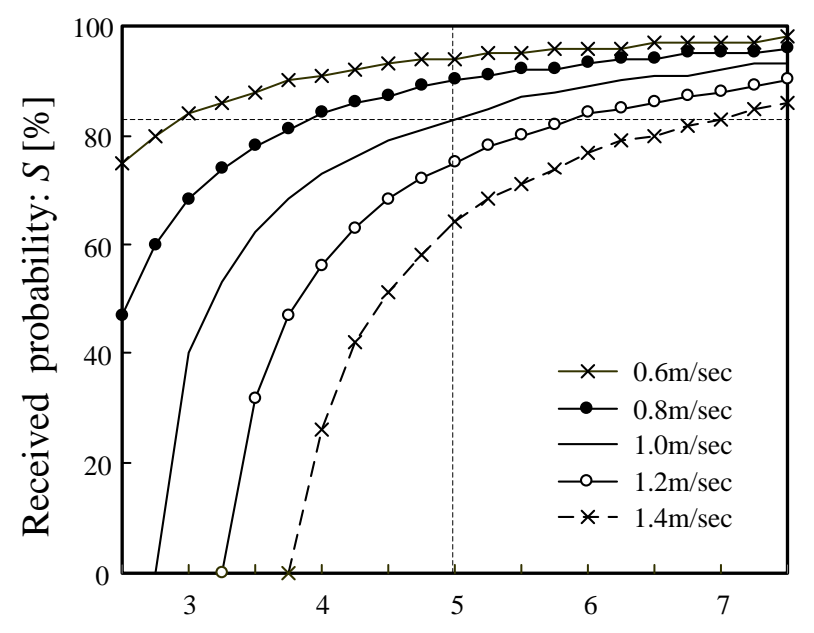

Communication area (Radius: $r$ ) [m]

Figure 11. Probability versus communication area.

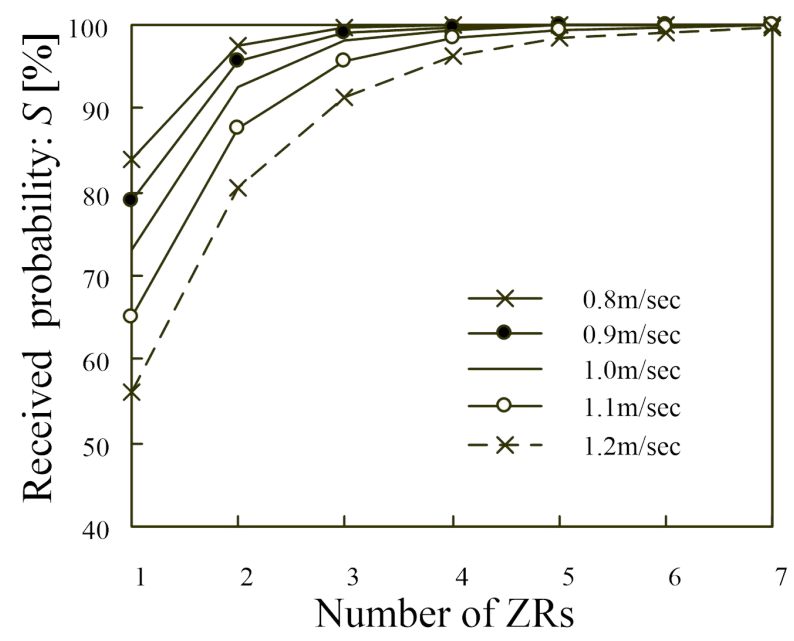

Figure 12. Probability versus the number of ZRs.

\section{Conclusion}

In this paper, experiments using actual ZigBee devices confirmed that the proposed scheme provided accurate location and vital health information by using sensor data. Moreover, the amount of the sensor data that could be transmitted when the terminal moved in communication area of ZigBee was confirmed by a numerical analysis. The results showed that in the proposed scheme, the ZigBee terminal could accurately detect the location. Over 99\% of the sensor data regarding vital health information was obtained when the ZigBee terminal passed through approximately four ZigBee routers.

\section{Acknowledgements}

Part of this work is supported by KAKENHI, a Grant-in-Aid for Scientific Research (C) (25420362 and 25330111), Telecommunication Advancement Foundation in 2014 and Hoso Bunka Foundation 2015.

\section{References}

[1] http://www.ntt.co.jp/csr\_e/2009report/safety/activity04.html

[2] Jawad, S.K., Jawad, S.K. and Al-Shagoor, B.E. (2008) A Multipurpose Child Tracking System Design and Implemen- 
tation. International Journal of Soft Computing Applications, 57-68.

[3] Khan, J.Y., Yuce, M.R., Bulger, G. and Harding, B. (2012) Wireless Body Area Network (WBAN) Design Techniques and Performance Evaluation. Journal of Medical Systems, 36, 1441-1457.

[4] IEEE 802.15.4-2006 Std. (2006) Wireless Medium Access Control and Physical Layer Specifications for Low-Rate Wireless Personal Area Networks. IEEE Computer Society.

[5] Blumenthal, J., Grossmann, R., Golatowski, F. and Timmermann, D. (2007) Weighted Centroid Localization in Zigbee-Based Sensor Networks. Proceedings of IEEE International Symposium on Intelligent Signal Processing, Alcala de Henares, 3-5 October 2007, 1-6. http://dx.doi.org/10.1109/WISP.2007.4447528

[6] Gomez, C., Boix, A. and Paradells, J. (2010) Impact of LQI-Based Routing Metrics on the Performance of a One-toOne Routing Protocol for IEEE 802.15.4 Multihop Networks. EURASIP Journal on Wireless Communication and Networking, 2010, Article ID 205407.

[7] Kaneko, N. and Nagasaka, Y. (2007) Position Estimation Method of Moving Objects by a Two-Point Positioning Technique with Active RFID Technology. Proc. Forum on Information Technology (FIT), Chukyo University, 5-7 September 2007, 285-286.

[8] IEEE 802.11WG (2007) IEEE Std. 802.11, Part 11: Wireless LAN Medium Access Control (MAC) and Physical Layer (PHY) Specifications. ISO/IEC 8802-11.

[9] Wi-Fi Alliance (2011) Wi-Fi in Health Care: The Solution for Growing Hospital Communication Needs. http://www.wi-fi.org/featured-topics

[10] Park, M.H., Kim, W.H., Lee, S.J. and Kim, H.C. (2012) GPS-Tag for Indoor Location Information and Additional User Information Providing. Proceedings of 14th International Conference on Advanced Communication Technology, PyeongChang, 19-22 Feburary 2012, 894-897.

[11] Wong, K.S., Ng, W.L., Chong, J.H., Ng, C.K., Sali, A. and Noordin, N.K. (2009) GPS Based Child Care System Using RSSI Technique. Proceedings of IEEE 9th Malaysia International Conference on Communications, Kuala Lumpur, 15-17 December 2009, 899-904.

[12] Jung, J., Ha, K., Lee, J., Kim, Y. and Kim, D. (2009) Wireless Body Area Network in a Ubiquitous Healthcare System for Physiological Signal Monitoring and Health Consulting. International Journal of Signal Processing, Image Processing and Pattern Recognition, Vol., 47-54.

[13] Kikuchi, A., Nakane, H. and Tsushima, H. (2011) Preliminary Study of Distance Needed to Achieve Constant Walking Speed and Step Length by Young Healthy Persons. Rigakuryoho Kagaku, 26, 647-650. http://dx.doi.org/10.1589/rika.26.647

[14] Hayes, T.L., Hagler, S., Austin, D., Kaye, J. and Pavel M. (2009) Unobtrusive Assessment of Walking Speed in the Home Using Inexpensive PIR Sensors. Proceedings of Annual International Conference of the IEEE Engineering in Medicine and Biology Society, Minneapolis, 3-6 September 2009, 7248-7251. http://dx.doi.org/10.1109/iembs.2009.5334746

[15] Vathsangam, H., Emken, A., Spruijt-Metz, D. and Sukhatme, G.S. (2010) Toward Free-Living Walking Speed Estimation Using Gaussian Process-Based Regression with On-Body Accelerometers and Gyroscopes. Proceedings of 4th International Conference on-NO PERMISSIONS Pervasive Computing Technologies for Healthcare (PervasiveHealth), Munich, 22-25 March 2010, 1-8.

[16] Rec. ITU-R P.1238-6 (2009) Propagation Data and Prediction Methods for the Planning of Indoor Radio Communication Systems and Radio Local Area Networks in the Frequency Range $900 \mathrm{MHz}$ to $100 \mathrm{GHz}$. ITU-R Recommendations.

[17] Hiraguri, T., Takase, H., Sugishita, T., Kimura, T., Aoyagi, M. and Nishimori, K. (2012) ZigBee-Based Scheme for Location Information and Data Transmission. IEICE Communications Express, 1, 72-77.

http://dx.doi.org/10.1587/comex.1.72 